\title{
How to teach interference and diffraction in a hurry
}

Henri Arsenault

Henri H. Arsenault, "How to teach interference and diffraction in a hurry," Proc. SPIE 3190, Fifth International Topical Meeting on Education and Training in Optics, (8 December 1997); doi: 10.1117/12.294372

Event: Fifth International Topical Meeting on Education and Training in Optics, 1997, Delft, Netherlands 


\title{
How to teach interference and diffraction in a hurry
}

\author{
Henri H. Arsenault \\ COPL, Université Laval. Québec, P.Q., Canada, G1K 7P4 \\ email: arseno@phy.ulaval.ca
}

\begin{abstract}
We introduce a new approach for teaching interference and diffraction, which expresses the Huyghens-Fresnel diffraction formula in terms of the observation plane. This makes it easier to obtain the expressions for Fraunhofer diffraction and for Youn's fringes, which appear as special cases, as well as the far-field condition. This allows interference and diffraction to be covered in a fraction of the classroom time usually required. Once the approximation is made, all waves are expressed in terms of their angular distance from the optical axis. The method may be applied to more complex cases like Newton's rings and diffraction by multiple slits.
\end{abstract}

Keywords: Optics education, interference, diffraction, teaching

\section{INTRODUCTION}

I have been teaching interference and diffraction over many years. Although these are relatively simple concepts, I have found that most of the effort in learning this material is spent on the approximations. Indeed, simple far-field diffraction from two sources yields the Young's fringes only after two approximations are made: the far-field approximation and the paraxial approximation. The former is the most difficult of the two. In diffraction, most textbooks have to restart from scratch with the approximations. In teaching interference and diffraction, whenever possible the emphasis should be on the Physics and not on the mathematical intricacies.

This paper assumes that the students are familiar with complex numbers. We restrict our analysis to one-dimension, and the extension to two dimensions is straightforward.

I have recently discovered that it is much simpler to make the approximations at the beginning. The whole question of this paper resides with how you write a wave exp(ik॰r). Because we are interested in what is going on in the observation plane, it is better to modify the diffraction integral to express the quantities in terms of the observation plane.

According to the Huyghens-Fresnel theory of diffraction, the diffracted field from an aperture with transmittance $f(r)$ is given by

$g(r)=\int_{\Omega} f\left(r_{1}\right) \frac{\exp \left(i \mathbf{k} \cdot \mathbf{r}_{1}\right)}{r_{1}} d r_{1}$

where $r_{1}$ is a vector from the diffracting plane to the point of observation, $f\left(r_{1}\right)$ is the transmittance of the diffracting pupil, and the integration is taken over the pupil $\Omega$.

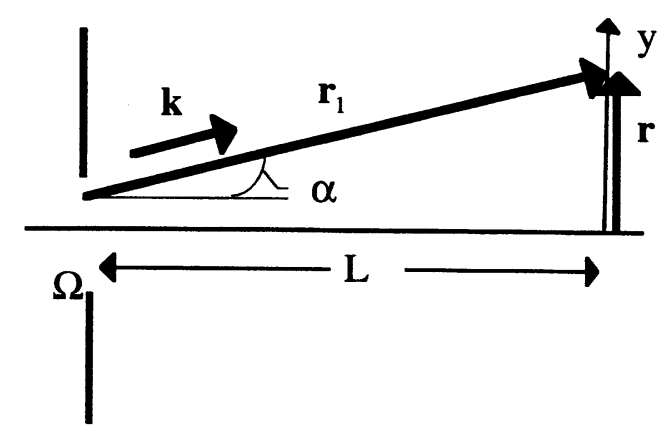

Figure 1: The geometry of diffraction 
Now from Fig. $1, \mathbf{r}_{1}=\mathbf{L}+\mathbf{r}$, so replacing $\mathrm{L}+\mathrm{r} \approx \mathrm{L}$ in the denominator

$$
g(r)=\frac{1}{L} \int f(r) \exp [i \mathbf{k} \cdot(\mathbf{L}+\mathbf{r})] d r_{1}
$$

Now $k \cdot r_{1}=k \cdot(L+r)=k(L \cos \alpha+y \sin \alpha)$ (see Fig. 1), so that the previous expression may be written:

$$
\left.g(y)=\int_{\alpha_{0}}^{\alpha_{1}} f \alpha\right) \exp (i k(L \cos \alpha+y \sin \alpha) d \alpha .
$$

Now using the approximation $\cos \alpha \approx 1$ and $\sin \alpha \approx \alpha$, this yields

$$
g(y)=e^{i k L} \int_{\alpha_{0}}^{\alpha_{1}} f(\alpha) e^{i k y \alpha} d \alpha
$$

where $y$ is the distance from the arbitrary origin along the line of observation, and $\alpha$ is the angle between the vector $\mathbf{k}$ and the optical axis. When the angle $\alpha$ is constant, this is often given as the expression for a plane wave, but it is valid for any paraxial wave (with a non-constant $\alpha$ ), and it represents the amplitude along the line of observation. Eq. (4) will be the starting point for our discussions on interference and diffraction. For a free aperture, $f(\alpha)=1$.

\section{THE FAR-FIELD CONDITION}

The far-field condition can easily be deduced from the approximations that we have made in passing from Eq. (3) to Eq. (4). The smallest term that we have neglected in setting $\cos \alpha \approx \alpha$ is $\mathrm{kL}^{2} / 2\left(\cos \alpha \approx 1+\alpha^{2} / 2+\ldots\right)$, and this term must satisfy the condition

$\mathrm{kL} \alpha^{2} / 2<\pi$,

and taking into account that $\alpha \approx \operatorname{tg} \alpha=d / L$, where $d$ is the aperture size, this condition yields the far-field condition

$d^{2} / \lambda<<1$

\section{TWO-BEAM INTERFERENCE}

Various kinds of two-beam interference may now be calculated from Eq. (4). The interference between two plane waves with angles $\alpha_{1}$ and $\alpha_{2}$ is

$$
\begin{aligned}
& \mathrm{g}(\mathrm{y})=\exp \left(\mathrm{iky} \alpha_{1}\right)+\exp \left(\mathrm{iky} \alpha_{2}\right) . \\
& \mathrm{g}(\mathrm{y})=\exp \left[\mathrm{iky}\left(\alpha_{1}+\alpha_{2}\right) / 2\right]\left[\exp \left[\mathrm{iky}\left(\alpha_{1}-\alpha_{2}\right) / 2\right]+\exp \left[-\mathrm{iky}\left(\alpha_{1}-\alpha_{2}\right) / 2\right]\right. \\
& \left.\mathrm{g}(\mathrm{y})=2 \exp \left[\mathrm{iky}\left(\alpha_{1}+\alpha_{2}\right) / 2\right] \cos \left[\mathrm{ky}\left(\alpha_{1}-\alpha_{2}\right) / 2\right)\right] .
\end{aligned}
$$

Now using the condition that $\alpha_{1}-\alpha_{2}=\theta$, where $\theta$ is the angle between the two wave vectors $k_{1}$ and $k_{2}$, the illumination on the observation plane is the classical expression, where $\mathrm{L}$ is the distance from the slits to the observation plane and $d$ is the distance between the slits:

$$
|g(y)|^{2} \approx 4 \cos ^{2}\left(\frac{k y \theta}{2}\right)=4 \cos ^{2}\left(\frac{\pi d y}{\lambda L}\right) \text {. }
$$




\section{FRAUNHOFER DIFFRACTION}

We now consider far-field diffraction for a single slit. The usual proofs usually begin from scratch, but we may build upon what we have just done for two-slit interference. Consider a slit of width d as illustrated in Fig. 2.

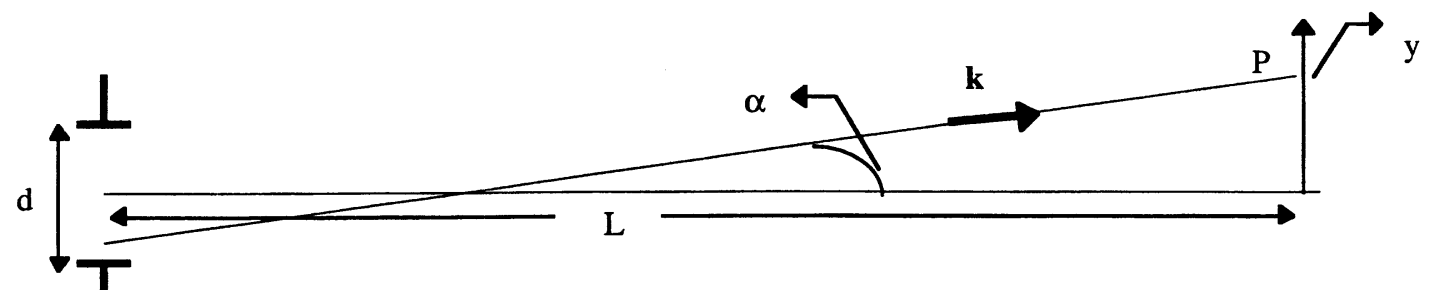

Figure 2: Fraunhofer diffraction from a single slit of width $d$ at a distance $L$

Now consider a wave in direction $\mathbf{k}$ from any point in the aperture to a point $\mathrm{P}$ at a distance $\mathrm{y}$ from the origin in the observation plane. As we have seen, the amplitude at point $P$ from this wave is approximately

$\mathrm{g}(\mathrm{y}) \approx \mathrm{A} \exp (\mathrm{iky} \alpha)$

where $A$ is the amplitude of the wave at point $P$.

Now the sum of all the waves coming from the aperture is

$$
g(y)=A \int_{\alpha_{0}}^{\alpha_{1}} \exp (i k y \alpha) d \alpha .
$$

where it is assumed that the amplitudes $A$ of all the incident waves are equal., and where $\alpha_{0}$ and $\alpha_{1}$ are the angles corresponding to the two extremities of the slit:

$$
\begin{aligned}
& \alpha_{0} \approx \tan \alpha_{0}=(\mathrm{y}+\mathrm{d} / 2) / \mathrm{L} \\
& \alpha_{1} \approx \tan \alpha_{1}=(\mathrm{y}-\mathrm{d} / 2) / \mathrm{L} .
\end{aligned}
$$

The integral of Eq. (11) yields

$$
\begin{aligned}
& g(y)=\left.A \frac{e^{i k y \alpha}}{i k y}\right|_{(y-d / 2) / L} ^{(y+d / 2) / L}=\frac{A}{i k y}\left[e^{i k y(y+d / 2) / L}-e^{i k y(y-d / 2) / L}\right] \\
& g(y)=\frac{A e^{\frac{i k y^{2}}{L}}}{i k y}\left[e^{i k y d / 2 L}-e^{-i k y d / 2 L}\right]
\end{aligned}
$$

which may be rearranged as

$$
g(y)=\frac{d A}{L} \exp \left(\frac{i k y^{2}}{L}\right) \frac{\sin (\pi \mathrm{yd} / \lambda \mathrm{L})}{\pi \mathrm{yd} / \lambda \mathrm{L}}
$$

So the illumination on the screen is equal to

$$
|g(y)|^{2}=\left(\frac{d A}{L}\right)^{2} \frac{\sin ^{2}(\pi y d / \lambda L)}{(\pi y d / \lambda L)^{2}}
$$

which is the classical expression for the diffraction figure from a slit. 


\section{NEWTON'S RINGS}

We now calculate the interference fringes from two sources one behind the other. The two sources are normally derived from the same source by means of reflecting surfaces. Let the two sources be at distances $\mathrm{L}_{1}$ and $\mathrm{L}_{2}$ as shown in Fig. 3 .

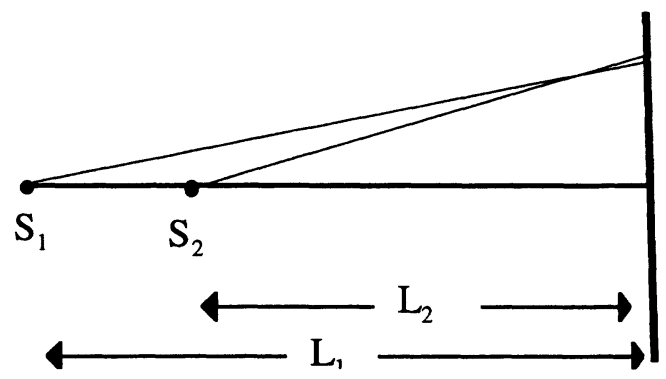

Figure 3: Interference between two sources one behind the other

The superposition from the two sources is again given by Eq. (6). This time, we have approximately

$\alpha_{1} \approx y / L_{1}, \alpha_{2} \approx y / L_{2}$

so that Eq. (6) yields for the illuminance on the observation plane

$\left.\lg (\mathrm{y})\right|^{2} \approx \cos ^{2}\left[\mathrm{ky}^{2}\left(1 / 2 \mathrm{~L}_{1}-1 / 2 \mathrm{~L}_{2}\right)\right.$,

which is the general expression for Newton's rings. When one of the sources is at infinity, say $\mathrm{L}_{2}=0$ and $\mathrm{L}_{1}=\mathrm{L}$, the expression yields the more common expression for Newton's rings:

$|g(y)|^{2} \approx \cos ^{2} \frac{2 \pi y^{2}}{\lambda L}$.

\section{CONCLUSION}

Let us review the advantages of the approach proposed here. 1) The expression for a wave exp(ik $\alpha$ ) is introduced early, and is used in a consistent manner for interference between two plane waves, for the far-field diffraction between two thin slits, and for Fraunhofer diffraction from a slit; 2) The demonstration of the diffraction of a slit is a direct generalization of the Young's interference between two slits, and is almost mathematically trivial.; 3 ) The far-field condition is easily derived from the approximations made; 4) the subjects can be covered in a fraction of the time that is usually required.

One might be tempted to say that the passage from $\exp (i k \sin \alpha)$ to $\exp (\mathrm{ik} \alpha)$ is a passage from an arbitrary wave to a plane wave, but this is not the case: a plane wave ensues when one assumes that $\alpha$ is constant, which is not necessarily assumed in the approximation.

The approach presented here is not limited to Fraunhofer diffraction and to the calculation of Young's fringes: for example, it can be used to calculate diffraction from multiple slits and the radiation pattern over $2 \pi$ from two or more sources. One should note, however that for electromagnetic waves, neglecting polarization could yield errors in the expression, for example, of the radiation pattern from a dipole. Finally we note that for the calculation of radiation patterns, the far-field condition still holds, but of course not the paraxial approximation. 